\title{
ANALYSER LES POLITIQUES PUBLIQUES INTERNATIONALES
}

Franck Petiteville et Andy Smith

Presses de Sciences Po | «Revue française de science politique »

2006/3 Vol. 56 | pages 357 à 366

ISSN 0035-2950

ISBN 2724630424

Article disponible en ligne à l'adresse :

https://www.cairn.info/revue-francaise-de-science-politique-2006-3-page-357.htm

Distribution électronique Cairn.info pour Presses de Sciences Po.

(C) Presses de Sciences Po. Tous droits réservés pour tous pays.

La reproduction ou représentation de cet article, notamment par photocopie, n'est autorisée que dans les limites des conditions générales d'utilisation du site ou, le cas échéant, des conditions générales de la licence souscrite par votre établissement. Toute autre reproduction ou représentation, en tout ou partie, sous quelque forme et de quelque manière que ce soit, est interdite sauf accord préalable et écrit de l'éditeur, en dehors des cas prévus par la législation en vigueur en France. Il est précisé que son stockage dans une base de données est également interdit. 


\title{
ANALYSER LES POLITIQUES PUBLIQUES INTERNATIONALES
}

\author{
FRANCK PETITEVILLE, ANDY SMITH
}

$\mathrm{E}$

n France et ailleurs dans le monde, l'analyse des politiques publiques et l'étude des relations internationales se sont constituées en sous-disciplines de la science politique sans beaucoup se croiser. Chacune a affirmé son identité sur la base de programmes de recherche relativement distincts, de ressources intellectuelles propres (concepts, théories, auteurs de référence ${ }^{1}$ ) et d'une certaine institutionnalisation disciplinaire (enseignements spécifiques, poste profilés et épreuves spécialisées à l'agrégation en France). Depuis quelques années toutefois, les passerelles se sont multipliées. L'appropriation d'objets de recherche « de » relations internationales par les sociologues de l'action publique est devenue plus fréquente et l'intérêt des internationalistes pour les concepts et méthodes de l'analyse des politiques publiques s'est affirmé dans un certain nombre de travaux. À la suite d'un colloque organisé par la section d'études internationales de l'Association française de science politique sur les «politiques publiques internationales » en avril 2005, ce numéro de la RFSP illustre ce rapprochement interdisciplinaire.

Dans cette introduction, nous chercherons à cerner quelques-uns des enjeux de la convergence entre analyse des politiques publiques et relations internationales. Plus précisément, à travers nos lectures et en croisant nos perspectives de recherche respectives, nous nous emploierons à défendre deux hypothèses. La première est que l'analyse des politiques et les relations internationales voient aujourd'hui leurs programmes de recherche se rapprocher tendanciellement autour des dynamiques d'européanisation, d'internationalisation et de transnationalisation de l'action publique. Notre seconde hypothèse est que les deux disciplines sont également susceptibles de rapprocher leur posture de recherche et leur méthodologie pour peu qu'elles acceptent les règles de la méthode sociologique, évolution certainement plus aboutie du côté de l'analyse des politiques publiques et qui peut ainsi contribuer à « sociologiser » davantage l'étude des relations internationales.

\section{LE RAPPROCHEMENT DES PROGRAMMES DE RECHERCHE}

L'institutionnalisation des relations internationales comme discipline académique s'est opérée après la première guerre mondiale aux États-Unis et au RoyaumeUni à partir de l'étude des relations inter-étatiques structurées par la diplomatie, la

1. Les deux dictionnaires récemment publiés en France dans les deux disciplines illustrent la spécificité des concepts propres à chacune d'entre elles. Cf. Marie-Claude Smouts, Dario Battistella, Pascal Vennesson, Dictionnaire des relations internationales, Paris, Dalloz, $2^{\mathrm{e}}$ édition, 2005, et Laurie Boussaguet, Sophie Jacquot, Pauline Ravinet (dir.), Dictionnaire des politiques publiques, Paris, Presses de Sciences Po, 2004. 
guerre, la sécurité collective, le droit international ${ }^{1}$. L'analyse des politiques publiques, elle, s'est développée dans les années 1960-1970 aux États-Unis à partir de la théorie des organisations et de l'étude de l'État providence occidental, de ses politiques et de ses mutations ${ }^{2}$.

Au-delà de leurs problématiques originelles respectives, les deux disciplines se sont attachées à analyser des milieux réputés très dissemblables. Les relations internationales se sont lancées, si l'on en croit les théoriciens réalistes ${ }^{3}$, dans l'étude d'un milieu fondamentalement anarchique, puisque dépourvu d'autorité mondiale susceptible de s'imposer à des États placés en situation « hobbesienne » d'insécurité collective. D'où la place qu'occupent traditionnellement le conflit et la guerre dans l'étude des relations internationales. L'analyse des politiques publiques est au contraire intervenue dans des sociétés pacifiées et normées, où les enjeux sont de fabriquer de «l'ordre dans des sociétés complexes ${ }^{4}$, de réguler les « désajustements » (entre secteurs, entre secteurs et territoires, entre acteurs et ressortissants) et d'adapter la régulation politique à la crise des États providence, au néolibéralisme, à l'intégration européenne et à la transformation des rapports centre-périphérie en général ${ }^{5}$. Dans cette perspective, la principale dynamique d'internationalisation investie par l'analyse des politiques publiques a été l'intégration européenne, à travers « l'européanisation des politiques publiques $»^{6}$. Le fait est que l'Union européenne a toutes les apparences d'un ordre régional « insularisé » par rapport au milieu international et en réalité beaucoup plus proche des ordres sociétaux : comme eux, c'est un espace social pacifié, institutionnalisé, normé, qui se prête (même si pas exclusivement) à des politiques publiques régulatoires ${ }^{7}$.

Il faut toutefois nuancer cette vision dichotomique des relations internationales et de l'analyse des politiques publiques reposant sur une opposition entre les milieux étudiés. L'analyse des politiques publiques, d'abord, peut revendiquer une longue tradition d'études de situations de conflit (sociaux, catégoriels, identitaires, etc. ${ }^{8}$ ), ce qui dément l'accusation fréquente selon laquelle elle constituerait une approche « lissée »

1. Sur la genèse des relations internationales comme discipline académique, cf. Dario Battistella, Théories des relations internationales, Paris, Presses de Sciences Po, 2003, p. 73-103.

2. Ceci est particulièrement net dans l'analyse du développement de la discipline que font Yves Mény et Jean-Claude Thoenig dans Politiques publiques, Paris, PUF, 1989. Cf. également le numéro spécial consacré aux «Approches nationales des politiques publiques », Revue française de science politique, 52 (1), février 2002.

3. Pour un premier aperçu de la théorie réaliste, cf. Dario Battistella, op. cit., p. 111-142.

4. Cf. Pierre Muller, «L'analyse cognitive des politiques publiques. Vers une sociologie politique de l'action publique », Revue française de science politique, 50 (2), avril 2000, p. 189-207, notamment p. 189.

5. Bruno Jobert, Jacques Commaille (dir.), Les métamorphoses de la régulation politique, Paris, LGDJ, 1999.

6. Pour un compte rendu synthétique des recherches auxquelles a donné lieu ce concept, cf. l'article de Claude Radaelli dans le Dictionnaire des politiques publiques, op. cit., p. 191200. Pour un état des lieux des orientations actuelles de la recherche, voir la contribution de Patrick Hassenteufel et Yves Surel, «Les politiques publiques en Europe » au colloque organisé par la revue Politique européenne, «Les études européennes en France : perspectives et développements », Paris, 21-22 octobre 2005.

7. Giandomenico Majone, La Communauté européenne, un État régulateur, Paris, Montchrestien, 1996 ; Andy Smith, Le gouvernement de l'Union européenne : une sociologie politique, Paris, LGDJ, 2004.

8. Ce point a été souligné il y a presque vingt ans dans Bruno Jobert, Pierre Muller, L'État en action. Politiques publiques et corporatismes, Paris, PUF, 1987. 
du politique, évacuant les rapports de force et donc inopérante dans les situations de conflit. Comme le souligne Yves Buchet de Neuilly, « l'existence de clivages n'est pas un facteur d'implosion d'une politique publique, mais une propriété même de tout espace de politique publique, et même un révélateur de son existence ${ }^{1}$.

S'agissant des relations internationales ensuite, on voit mal en quoi la conflictualité du milieu international serait en soi rédhibitoire à des études de politique publique. On sait depuis Allison (et même un peu avant) qu'une sociologie fine de la décision peut tout à fait s'appliquer aux politiques étrangères, y compris dans les domaine dits de high politics et de hard security et en situation de crise ${ }^{2}$. Par ailleurs, le paradigme de l'anarchie du milieu international a été abondamment discuté et amendé par les théoriciens non réalistes, libéraux en particulier, qui ont insisté sur les effets de l'interdépendance économique entre États (théorie du « doux commerce »), de la dissémination internationale des normes démocratiques (théorie de la paix démocratique), ainsi que sur le rôle des organisations internationales et du droit international dans la domestication de l'anarchie internationale ${ }^{3}$. Les réalistes eux-mêmes ont pour la plupart admis que l'anarchie peut être tempérée par l'existence de régimes internationaux ${ }^{4}$.

Or, sous l'effet d'un encadrement normatif croissant des relations internationales dans l'après-guerre froide ${ }^{5}$, certains régimes internationaux apparaissent à ce point structurés aujourd'hui qu'ils peuvent être analysés comme des « ordres internationaux sectoriels » ou, pour parler le langage des politiques publiques, comme des secteurs internationaux où se déploie alors de l'action publique multilatérale : régulation du commerce international à l'OMC, mais aussi régime de sécurité instauré par les traités de désarmement, régime international de l'environnement en gestation, etc. ${ }^{6}$ Comme nous le verrons plus loin, la multiplication des régimes internationaux et l'institutionnalisation des relations internationales dont ils sont porteurs constituent des facteurs particulièrement propices à des approches de sociologie de l'action publique. Au total, les milieux étudiés par les relations internationales et par l'analyse des politiques ne sont pas aussi éloignés et dissemblables qu'on pourrait le penser. Restent les postures de recherche et les méthodologies.

1. Yves Buchet de Neuilly, «Une Europe sans voix : les conditions du recours à la PESC », dans Damien Helly, Franck Petiteville (dir.), L'Union européenne, acteur international, Paris, L'Harmattan, 2005, p. 75-90, dont p. 76.

2. Graham Allison, Philip Zelikow, Essence of Decision, Explaining the Cuban Missile Crisis, New York, Longman, $2^{\circ}$ éd., 1999.

3. Pour une première approche du courant libéral, cf. Dario Battistella, op. cit, p. 143-173.

4. Rappelons que la notion de régime international suppose l'existence de «principes», «normes », « règles » et «procédures » qui structurent un domaine de coopération internationale. Cf. l'ouvrage classique dirigé par Stephen Krasner, International Regimes, Ithaca, Cornell University Press, 1983, et, pour un débat actualisé, Andreas Hasenclaver et al, Theories of International Regimes, Cambridge, Cambridge University Press, 1997.

5. Guillaume Devin, Claude Gauthier, «Mondialisation et droit international public: entre réalismes juridique et politique », dans Josepha Laroche (dir.), Mondialisation et gouvernance mondiale, Paris, PUF, 2003, p. 251-262.

6. Cf. par exemple et, respectivement, sur l'OMC : Michel Rainelli, L'OMC, Paris, La Découverte, $7^{\mathrm{c}}$ éd., 2004 (Repères); sur le désarmement : Jozef Goldblat, Arms Control, the New Guide to Negotiations and Agreements, Londres, Sage, 2002, et Jean Klein, « Vingt ans de négociations sur le désarmement et la maîtrise des armements », Politique étrangère, 3, 1999, p. 663-678; sur le régime international de l'environnement : Philippe Le Prestre, Protection de l'environnement et relations internationales: les défis de l'écopolitique mondiale, Paris, Armand Colin, 2005, ainsi que Raphaël Romi, Droit international et européen de l'environnement, Paris, Montchrestien, 2005. 


\section{LE RAPPROCHEMENT DES POSTURES ET MÉTHODES DE RECHERCHE}

Plusieurs différences notables peuvent être relevées quant aux postures et méthodes de recherche des deux disciplines. Les relations internationales s'affichent volontiers comme une science de la «synthèse » et la sociologie de l'action publique comme une science « analytique ». Cette opposition renvoie non pas tant à une différence de programmes de recherche qu'à une différence de cadrage et de niveau du questionnement. Dans sa définition des politiques publiques, Jean-Claude Thoenig écrit que celles-ci sont des problèmes « analytiquement construits » ${ }^{1}$. Cette démarche analytique (qui conduit le chercheur à reconstituer une politique publique par l'identification de ses acteurs et ressortissants, l'élucidation de leur cadre cognitif, la reconstitution des différentes phases de l'action publique, etc.) ${ }^{2}$ fait corps en quelque sorte avec la discipline elle-même au point d'avoir été inscrite dans son label scientifique d'origine (on dit «l'analyse des politiques publiques » comme on ne dit pas « l'analyse des relations internationales »).

L'affichage des relations internationales comme «science de synthèse » s'illustre, elle, dans la propension de la discipline à produire des théories parfois très globalisantes (la « structure » du système international comme fonction de la distribution internationale de la puissance chez Waltz ${ }^{3}$, l'avènement d'une «gouvernance mondiale » à l'ère de la globalisation chez Rosenau ${ }^{4}$, etc.). Même dans leur dimension plus sociologique, les recherches menées en relations internationales sont souvent portées à l'agrégation et à la hiérarchisation d'une infinitude de variables. Établir par exemple, dans le cadre des programmes de recherche sur la paix démocratique, des corrélations entre le nombre de régimes démocratiques dans le monde et les probabilités de guerre et de paix peut conduire les chercheurs à travailler sur des séries statistiques longues portant sur des dizaines de conflits étalés sur plus d'un siècle ${ }^{5}$. De même, analyser les négociations multilatérales qui se jouent à 150 États à l'OMC et à 190 États pour le traité de non-prolifération nucléaire (TNP) impose aussi, nécessairement, la synthèse.

Qu'il y ait une part de vrai dans une plus forte inclination des relations internationales à la synthèse n'oblige pas pour autant à la caricature. L'étude des relations internationales ne se réduit pas à la confontation de méta-théories, ni à des synthèses globales. La démarche sociologique en relations internationales, qui constitue certainement un trait particulièrement marqué dans l'histoire de la discipline en France, a conduit à de nombreuses recherches, au plus près des acteurs, sur la manière dont se construisent politiques étrangères, politiques de défense, politiques des organisations

1. Dans Laurie Boussaguet, Sophie Jacquot, Pauline Ravinet (dir.), op. cit., p. 326.

2. Pour une illustration de cette démarche de construction de l'objet de recherche, cf. le chapitre 5, "Éléments pour une stratégie de recherche », du petit livre classique de Pierre Muller, Politiques publiques, Paris, PUF, 4 éd., 2000.

3. Kenneth Waltz, Theory of International Politics, New York, MacGraw-Hill, 1979.

4. James N. Rosenau, Ernst-Otto Czempiel, Governance without Governement : Order and Change in World Politics, Cambridge, Cambridge University Press, 1992.

5. Cf. l'étude classique (dont la méthodologie a été souvent reprise depuis) de Melvin Small, The Wages of War (1815-1865) : A Statistical Handbook, New York, John Wiley, 1972. Sur cette méthodologie du programme de recherche sur la paix démocratique, cf. Pascal Vennesson, «Renaissante ou obsolète ? La guerre aujourd'hui », Revue française de science politique, 48 (3-4), juin-août 1998, p. 515-534. 
internationales, etc. ${ }^{1}$ Inversement, l'analyse des politiques publiques n'est pas toujours «micro-empiriste » : les débats qui animent la discipline - sur la rationalité et l'irrationalité de l'action publique, la «perte de centralité » de l'État, la gouvernance des sociétés post-industrielles ou le « sens » de l'intégration européenne - montrent que la sociologie de l'action publique peut être tout aussi portée aux « grands débats » que les relations internationales ${ }^{2}$. La dichotomie « relations internationales, science de synthèse $» / \ll$ politiques publiques, science analytique » doit donc là encore être relativisée.

La construction de l'objet de recherche n'oppose pas non plus de manière radicale les deux disciplines. Pour l'analyste des politiques publiques, le «moment» de la recherche consacré à la construction de l'objet est considéré comme essentiel pour éviter le piège du nominalisme, « tant pour l'analyse des acteurs d'une politique publique que pour une politique publique en tant que telle $»^{3}$. En relations internationales, la tradition est davantage positiviste, due à la nature des objets étudiés (des activités sociales comme la diplomatie ou la guerre ne nécessitent pas de lourdes constructions de l'objet) et à l'héritage épistémologique de l'histoire diplomatique et du droit international dans la structuration de la discipline. Reste que les concepts qui sont au cœur des relations internationales (anarchie, intérêt national, sécurité, puissance) font l'objet d'un travail régulier de débats et de « déconstruction » depuis trente ans ${ }^{4}$. Par ailleurs, les internationalistes contemporains sont aujourd'hui systématiquement conduits à déconstruire les thèmes qui saturent le discours politico-médiatique sur les relations internationales : « guerre contre le terrorisme » ${ }^{5}$, « fin » des États dans la mondialisation ${ }^{6}$, « irrationalité ethnique » et « chocs identitaires » des conflits de l'après-guerre froide ${ }^{7}$, etc. La distance critique par rapport à l'objet de recherche est donc une précaution commune aux relations internationales comme à l'analyse des politiques publiques.

Enfin, les divergences méthodologiques souvent évoquées entre relations internationales et analyse des politiques publiques ne sont pas non plus aussi éclatantes qu'on veut bien le dire. La méthodologie éprouvée pour étudier les politiques publiques

1. On renverra ici aux multiples travaux d'auteurs comme Raymond Aron, Marcel Merle, Bertrand Badie, Marie-Claude Smouts, Samy Cohen, Marie-Christine Kessler, Guillaume Devin, Didier Bigo, Pascal Vennesson, Frédéric Charillon.

2. Cette montée en généralité dans les débats de politiques publiques apparaît assez bien dans les manuels récents de Jean-Pierre Gaudin, Sociologie de l'action publique, Paris, Presses de Sciences Po, 2002, et de Gilles Massardier, Politiques et action publiques, Paris, Armand Colin, 2003.

3. Patrick Hassenteufel, «Deux ou trois choses que je sais d'elle : remarques à propos d'expériences de comparaisons européennes », dans CURAPP (dir.), Les méthodes au concret, Paris, PUF, 2000, p. 110.

4. Après le débat réalistes/libéraux qui a marqué les années 1970-80, les années 1990 ont été dominées par la critique des constructivistes, qui ont cherché à montrer que ces notions d'inspiration réaliste n'étaient pas intangibles, mais historiquement et sociologiquement construites par des représentations collectives. Pour une synthèse sur l'approche constructiviste en relations internationales, cf. Dario Battistella, op. cit., p. 267-298.

5. Gilles Andréani, « The "War on Terror": Good Cause, Wrong Concept », Survival, 46 (4), hiver 2004-2005, p. 31-50.

6. Pour une synthèse des contre-arguments sur ce thème, cf. Samy Cohen, La résistance des États, les démocraties face aux défis de la mondialisation, Paris, Seuil, 2003.

7. Stathis N. Kalyvas, «Les guerres civiles après la guerre froide», dans Pierre Hassner, Roland Marchal (dir.), Guerres et sociétés. État et violence après la guerre froide, Paris, Karthala, 2003, p. 107-135. Cf. également Jean-François Bayart, L'illusion identitaire, Paris, Fayard, 1996. 
(enquêtes de terrain pour accéder aux acteurs et aux ressortissants des politiques) est parfois plus difficilement transposable à l'étude de certaines politiques internationales (la politique américaine actuelle en Irak, par exemple). Pour autant, les obstacles à la recherche empirique dans les relations internationales, pour être parfois imposants, ne sont pas insurmontables (fuites ou témoignages d'acteurs prééminents ${ }^{1}$, accès différé aux acteurs, ouverture d'archives, etc. ${ }^{2}$ ). Inversement, les difficultés méthodologiques peuvent être également importantes en sociologie politique «interniste » (sur la corruption, par exemple) et en sociologie de l'action publique (sur les politiques de défense). Si l'on met donc de côté la question du coût des déplacements du chercheur, il est certainement plus utile de poser la question des difficultés méthodologiques de la recherche comme un problème de toute sociologie du politique.

Faut-il alors conclure que rien ne s'oppose à une convergence plus aboutie des deux disciplines ? Convergence n'est certes pas fusion. De nombreux internationalistes continueront à ignorer les concepts et méthodes de l'analyse des politiques publiques pour étudier la problématique de la guerre et de la paix. De nombreux sociologues de l'action publique pourront considérer comme secondaires des synthèses de relations internationales sans impact sur la politique publique et les problèmes d'action publique qu'ils étudient. D'une manière générale, les problématiques qui ont respectivement structuré les programmes de recherche des relations internationales et de l'analyse des politiques publiques (la guerre, la diplomatie, le multilatéralisme dans un cas, les mutations de l'État providence et la régulation des sociétés post-industrielles dans l'autre) ont de bonnes raisons de continuer à produire leurs effets dans l'avenir, pour maintenir les deux disciplines dans leurs vocations et délimitations respectives.

Reste que l'analyse des politiques publiques et les relations internationales ont de bonnes chances de se rencontrer de plus en plus fréquemment et de se féconder mutuellement autour de l'analyse des processus d'institutionnalisation des relations internationales en général et de la production croissante de «politiques publiques internationales » en particulier.

\section{LES POLITIQUES INTERNATIONALES : DES POLITIQUES PUBLIQUES COMME LES AUTRES ?}

Si l'on entend par «politiques publiques internationales » l'ensemble des programmes d'action revendiqués par des autorités publiques ayant pour objet de produire des effets dépassant le cadre d'un territoire stato-national, on peut distinguer deux types de politiques publiques internationales : les « politiques étrangères »d'une part, relevant d'une autorité publique centrale (les États, mais aussi, par extension, une organisation relativement intégrée comme l'Union européenne aujourd'hui), et,

1. Cf. par exemple sur l'affaire des « armes de destruction massive » qu'aurait accumulées le régime de Saddam Hussein, le témoignage de Hans Blix, Irak, les armes introuvables, Paris, Fayard, 2004.

2. Cf. par exemple la publication des enregistrements des débats du comité exécutif du Conseil national de sécurité américain qui a permis, dans l'affaire des missiles de Cuba, de réévaluer à la hausse le rôle personnel de Kennedy dans la gestion de la crise par rapport à la thèse bureaucratique privilégiée par Allison: Ernest May, Philip Zelikow, The Kennedy Tapes: Inside the White House during the Cuban Missile Crisis, Cambridge (Mass.), The Belknap Press of Harvard University Press, 1997. Cf. également le renouveau de la recherche sur la guerre froide occasionné par l'ouverture des archives du Pacte de Varsovie. 
d'autre part, les politiques publiques multilatérales produites par (ou dans le cadre) d'organisations internationales.

L'analyse des politiques publiques est présente de longue date dans l'étude des politiques étrangères. Elle constitue la tradition dite de la Foreign Policy Analysis. C'est néanmoins une tradition qu'il faut fréquemment réactiver en France, où elle a toujours eu moins d'adeptes que dans le monde anglo-saxon ${ }^{1}$. C'est par ailleurs une tradition qu'il faut étendre à l'étude de politiques étrangères post-nationales comme la « politique étrangère » de l'Union européenne. Un auteur comme Brian White plaide ainsi pour l'adaptabilité du cadre méthodologique et conceptuel de la Foreign Policy Analysis à la politique internationale de l'Union européenne, en réfutant le stato-centrisme présumé de la Foreign Policy Analysis ${ }^{2}$. Mais nombreux sont les auteurs qui, sans revendiquer explicitement l'affiliation à la Foreign policy Analysis, mènent sur les politiques externes de l'UE des recherches qui relèvent de ce type de démarche, combinant enquête de terrain auprès des acteurs et mise à jour des processus bureaucratiques et institutionnels par lesquels s'élabore la politique étrangère européenne ${ }^{3}$.

Dans l'étude de « la genèse de la coopération franco-allemande » qu'il présente dans ce numéro de la RFSP, Mathias Delori combine ainsi les apports de la Foreign Policy Analysis, le two-level game de Putnam, l'approche cognitive des politiques publiques et le concept de path dependance pour revisiter la naissance de cette coopération. Il n'invalide pas alors les approches classiques de l'histoire diplomatique (d'inspiration réaliste) selon lesquelles le rôle personnel de De Gaulle et Adenauer et les configurations stratégiques (européennes et internationales) dans lesquelles se trouvaient alors prises la France et l'Allemagne ont largement déterminé la forme et le contenu de la réconciliation franco-allemande. Mais il met à jour le rôle joué par les administrations des ministères des Affaires étrangères et les groupes associatifs d'après-guerre dans la structuration de la coopération franco-allemande autour d'une dimension culturelle qui sera longtemps prééminente. Il souligne aussi le caractère à la fois contingent, aléatoire et déterminant pour l'avenir (défense) de la coopération scellée autour du traité de l'Élysée en 1963.

L'étude des politiques publiques multilatérales produites par des organisations internationales constitue un deuxième champ prometteur pour le croisement entre relations internationales et politiques publiques. La globalisation, dont les sociologues de l'action publique reconnaissent à quel point elle pèse sur l'élaboration des politiques publiques ${ }^{4}$, accroît en effet les interdépendances entre États et suscite alors de leur part des réponses collectives et coopératives. Il en résulte une "multilatéralisation » de l'action publique qui vise à produire une lecture commune de phénomènes

1. Cf. notamment en France les travaux de Samy Cohen : La monarchie nucléaire: les coulisses de la politique étrangère sous la Cinquième République, Paris, Hachette, 1986 ; ainsi que «Décision, pouvoir et rationalité dans la politique étrangère » dans Marie-Claude Smouts (dir.), Les nouvelles relations internationales, Paris, Presses de Sciences Po, 1998, p. 75-101.

2. Cf. Brian White, Understanding European Foreign Policy, Basingstoke, Palgrave, 2001, et, plus récemment, « Foreign Policy Analysis and the New Europe », dans Walter Carlsnaes, Helen Sjursen, Brian White (eds), Contemporary European Foreign Policy, Londres, Sage, 2004, p. 11-31.

3. Cf. sur des registres d'analyse différents, Yves Buchet de Neuilly, L'Europe de la politique étrangère, Paris, Economica, 2005, et Damien Helly, «Les modes d'action extérieure de l'Union européenne dans le Caucase du Sud », Revue française de science politique, 55 (2), avril 2005, p. 243-268.

4. Cf. l'article précité de Pierre Muller, «Vers une sociologie politique...», art. cité, notamment p. 203-205. 
« globaux » (le changement climatique, les « risques systémiques » du système financier international, etc.), à adopter des normes de régulation multisectorielles (commerciale, financière, environnementale, sanitaire, etc.), voire à mettre en place des processus injonctifs à l'endroit des États (décisions de l'organe de règlements de différends de l'OMC, etc.). Dans la plupart des cas, cette recherche de "gouvernance » de la globalisation a entraîné une revitalisation des grandes organisations internationales ${ }^{1}$.

Dans cette perspective, des sociologues et analystes de politiques publiques de plus en plus nombreux s'intéressent aujourd'hui à ces organisations internationales et au rôle qu'elles jouent dans la « transnationalisation de l'action publique $»^{2}$ : rôle de la Banque mondiale dans l'imposition d'un «modèle » de restructuration des systèmes de protection sociale à l'échelle mondiale ${ }^{3}$, rôle de l'OIT dans la diffusion internationale de normes sociales ${ }^{4}$, etc. D'une manière générale, la connaissance des organisations internationales et de l'action publique multilatérale qu'elles promeuvent ne peut que tirer bénéfice d'études mobilisant de manière systématique les méthodes et concepts de l'analyse des politiques publiques. Comment ne pas voir ainsi l'intérêt à analyser la nonréforme de l'ONU en terme d'approche organisationnelle et de path dependence, l'OMC comme forum de production de normes et d'expertise dans la régulation du commerce, l'influence des institutions de Breeton Woods sur les États en développement en termes de transfert de modèles, la gestion du changement climatique par l'ONU en termes de constitution de communauté épistémique et d'expertise internationale ?

Deux articles publiés dans le cadre de ce numéro explorent à ce titre des coopérations internationales encore peu connues. Frédérique Channac s'intéresse au cas des «migrations internationales » et montre comment la gestion d'un enjeu interdépendant par excellence ( 175 millions de migrants dans le monde) amorce une coopération intergouvernementale croissante dans un domaine pourtant particulièrement sensible, généralement assimilé aux prérogatives de l'État souverain. La coopération internationale s'institutionnalise pourtant, avec la réforme de l'Organisation internationale pour les migrations, la réflexion sur la création de nouvelles structures et la coopération intergouvernementale consultative et informelle qui se développe à l'échelle régionale. L'auteur utilise notamment les concepts de policy transfer et de learning process pour éclairer les modalités de cette coopération non contraignante, essentiellement vouée à la circulation de l'information, à l'acquisition d'un langage commun sur les migrations et au transfert de modes opératoires (notamment d'Europe vers d'autres régions du monde). On retrouve de manière exemplaire dans ce cas l'enchaînement de causalités exposé précédemment : globalisation/interdépendance/multilatéralisme/institutionnalisation de la coopération/action publique multilatérale (en gestation dans le cas exposé).

Enfin, dans leur article sur «les politiques internationales de normalisation», François-Xavier Dudouet, Delphine Mercier et Antoine Vion abordent les normes

1. Pour une illustration de cette interaction entre globalisation et multilatéralisme, cf. Jean-Christophe Graz, La gouvernance de la mondialisation, Paris, La Découverte, 2004 ; Elie Cohen, L'ordre économique mondial, essai sur les autorités de régulation, Paris, Fayard, 2001.

2. Patrick Hassenteufel, « De la comparaison internationale à la comparaison transnationale. Les déplacements de la construction d'objets comparatifs en matière de politiques publiques », Revue française de science politique, 55 (1), février 2005, p. 113-132.

3. Bruno Palier, Louis-Charles Viossat (dir.), Politiques sociales et mondialisation, Paris, Futuribles, 2001.

4. Jean-Michel Bonvin, L'organisation internationale du travail : étude sur une agence productrice de normes, Paris, PUF, 1998. 
relatives à la qualité, à la sécurité et la compatibilité des biens et services à l'échelle internationale qui s'appliquent en particulier à l'apparition de nouveaux objets de consommation, de nouvelles méthodes de production et de transports et de nouveaux marchés. Cette contribution à l'étude des modalités concrètes de ce que l'on appelle souvent de manière abstraite la «régulation de la globalisation» est aussi une contribution au débat sur les normes, qui intéresse autant l'analyse des politiques publiques ${ }^{1}$ que les relations internationales ${ }^{2}$. Les auteurs mettent notamment à jour trois modes d'élaboration des normes qu'ils étudient : l'imposition par la puissance financière et commerciale (Microsoft et ses logiciels, par exemple), la négociation entre firmes concurrentes dont les acteurs publics ne sont pas éloignés (normes de qualité « ISO », normes de production « bio » ou encore norme «GSM » pour le marché de la téléphonie mobile), et la coopération intergouvernementale aboutissant à des réglementations internationales et/ou européennes (médicaments, AOC, normes européennes de sécurité des transports, etc.).

Les trois articles qui suivent illustrent donc, chacun à leur manière, la contiguïté programmatique, conceptuelle et méthodologique qui existe aujourd'hui entre sociologie de l'action publique et relations internationales dans l'étude des politiques publiques internationales. À notre sens, cette contiguïté impose une plus grande pratique de la transdisciplinarité. Celle-ci peut être bénéfique à chacune des deux disciplines : elle ne peut qu'inciter les internationalistes à « sociologiser» davantage leur étude de la politique internationale et elle ouvre à l'analyse des politiques publiques le champ insuffisamment exploré de l'action publique internationale ${ }^{3}$.

Franck Petiteville est maître de conférences de science politique à l'université René Descartes-Paris V et chercheur associé au CERI. Il a récemment publié : (en codirection avec Damien Helly) L'Union européenne, acteur international, Paris, L'Harmattan, 2005 ; «L'hégémonie est-elle soluble dans le multilatéralisme ? Le cas de l'OMC», Critique internationale, 22, janvier 2004, p. 63-76. Il va publier: L'Europe et le monde, la politique internationale de l'Union européenne, Paris, Presses de Sciences Po, à paraître en 2006. Ses recherches actuelles portent sur les politiques externes de l'Union européenne (<f.petiteville@wanadoo.fr>).

Andy Smith est directeur de recherche à la Fondation nationale des sciences politiques et travaille au laboratoire CNRS-CERVL à Bordeaux. Spécialiste de l'intégration européenne, il a notamment publié Le gouvernement de l'Union européenne, Paris, LGDG, 2004, et a dirigé Politics and the European Commission, Londres, Routledge, 2004. Il étudie actuellement la dimension politique de la régulation du commerce international en général et la manière dont elle oriente les secteurs viticole et agroalimentaire en particulier (<a.smith@ @sciencespobordeaux.fr >).

1. Cf. l'article de Pierre Lascoumes dans Laurie Boussaguet, Sophie Jacquot, Pauline Ravinet (dir.), op. cit., p. 298-304.

2. Cf. la table ronde organisée par Yves Schemeil et Wolf Eberwein, «L'énonciation des normes internationales », Congrès de Lyon de l'Association française de science politique, septembre 2005.

3. Nous remercions Pierre Favre, Guillaume Devin et Jean Leca pour leurs commentaires sur des versions antérieures de ce texte. 


\section{Franck Petiteville, Andy Smith}

\section{RÉSUMÉ/ABSTRACT}

\section{ANALYSER LES POLITIQUES PUBLIQUES INTERNATIONALES}

L'analyse des politiques et les relations internationales voient aujourd'hui leurs programmes de recherche se rapprocher, obligeant à remettre en question la distance épistémologique entre ces deux parties des sciences du politique. Deux séries de dynamiques observées dans chaque « sous-discipline » concourent à leur convergence croissante : l'européanisation, l'internationalisation et la transnationalisation de l'action publique ; l'institutionnalisation des relations internationales, la consolidation des régimes internationaux et le renouveau du multilatéralisme. Cet article défend l'idée que les deux "sous-disciplines » peuvent ainsi se féconder mutuellement autour de l'étude des "politiques publiques internationales », qu'il s'agisse des politiques étrangères des États ou de l'Union européenne, ou de «l'action publique multilatérale » produite par les grandes organisations internationales.

\section{STUDYING INTERNATIONAL POLICY-MAKING}

Currently, the research agendas of Public Policy Analysis and International Relations are converging and thus have begun to challenge the epistemplogical distance between these two parts of political science. Two trends that are evident in both are accelerating this process : the Europeanisation, internationalisation and transnationalisation of public action; the institutionnalisation of international relations, the consolidation of international regimes and the reinvention of multilateralism. This article argues that both of these " sub-disciplines » can benefit from the cross-ferilizations which can arise from studying "international public policies », be they the foreign policy of states or the European Union or the "multilateral public action " produced by the major international organizations. 